\title{
Estado y bienestar rural argentino en la primera mitad del siglo XX. Un problema historiográfico*
}

María José Ortiz Bergia

\begin{abstract}
Profesora de la Universidad Nacional de Córdoba (Argentina). Correo electrónico: ortizbergia.mj@ gmail.com. La autora es doctora en Historia de la Universidad Nacional de Córdoba (Argentina). Entre sus publicaciones recientes tenemos: "La centralización estatal en la Argentina y el sistema de salud público cordobés en la primera mitad del siglo XX", Trabajos y Comunicaciones, $\mathrm{N}^{\circ} 44$ (2016) y "Asociaciones vecinales en la posguerra: nuevas demandas y prestaciones sociales, ciudad de Córdoba", Revista de Historia Americana y Argentina, Vol. 51 Nº 2 (2016). Entre sus temas de interés están historia social Argentina, historia del Estado, políticas públicas, bienestar y metodología de la investigación histórica.
\end{abstract}

\section{Alejandra Salomón}

Profesora de la Universidad Nacional de Quilmes-UNQ (Argentina) e Investigadora del Consejo Nacional de Investigaciones Científicas y Técnicas-CONICET (Argentina). Correo electrónico: alejandralaurasalomon@gmail.com. La autora es doctora en Ciencias Sociales y Humanas de la Universidad Nacional de Quilmes (Argentina). Entre sus publicaciones recientes tenemos: en coautoría "Brechas del bienestar: el problema de la vivienda rural argentina entre las décadas de 1930 y 1950". Revista Trashumante No 10 (2017) y "La electrificación rural en la agenda pública del gobierno de la provincia de Buenos Aires. Argentina, 1946-1955”, en Revista de Historia Iberoamericana, Vol. 10 No. 1 (2017). Entre sus temas de interés están Historia socio-política argentina, políticas públicas, bienestar rural y desarrollo local.

Recibido: 29 de agosto de 2016

Aprobado: 2 de marzo de 2017

Modificado: 15 marzo de 2017

Artículo de investigación científica

DOI: http://dx.doi.org/10.15648/hc.31.2017.2

* $\quad$ Este artículo forma parte del proyecto: "Peronismo, políticas públicas y bienestar rural en la provincia de Buenos Aires (1946-1955)" financiado por el Consejo Nacional de Investigaciones Científicas y Técnicas-CONICET (Argentina). y el proyecto "Grupos sociales, diferenciación y desigualdad social en una sociedad heterogénea y cambiante, 1870-1995” financiado por la Universidad Católica de Córdoba (Argentina).

Esta publicación está bajo una licencia Creative Commons Reconocimiento-NoComercial 4.0 
Estado y bienestar rural argentino en la primera mitad del siglo XX. Un problema historiográfico

\begin{abstract}
Resumen
El propósito es avanzar en la construcción del bienestar rural estatal como objeto de interés historiográfico. En tal dirección, se realiza un análisis crítico del bienestar rural argentino en perspectiva histórica, circunscrito a la primera mitad del siglo XX. Para ello, primero, se exploran los aportes historiográficos producidos sobre esta temática, sus posibilidades analíticas y algunos vacíos subsistentes. A continuación, se debate sobre los usos de lo rural en la comprensión histórica de las políticas sociales. Finalmente, se resumen algunos avances realizados en la dilucidación de las experiencias históricas del bienestar rural estatal en la Argentina que pueden ser útiles para una discusión en ciernes.
\end{abstract}

Palabras clave: Bienestar rural, políticas públicas, Estado, historiografía social.

State and rural Argentine welfare in the first half of the 20th century. A historiographic problem

\begin{abstract}
The purpose is to progress on the construction of the state rural welfare as an object of historiographic interest. In this sense, a critical analysis of the Argentine rural welfare is carried out from a historical perspective, circumscribed to the first half of the twentieth century. To do so, firstly, the historiographic contributions produced on this subject are explored, as well as its analytical possibilities and some remaining gaps. Secondly, the uses of the rural in the historical understanding of social policies are discussed. Finally, it is made a summary of some advances in elucidating the historical experiences of state rural welfare in Argentina that may be useful for a budding discussion.
\end{abstract}

Keywords: Rural welfare, public policies, State, social historiography.

Estado e bem-estar rural argentino na primeira metade do século XX. Um problema historiográfico

\title{
Resumo
}

O propósito é avançar na construção do bem-estar rural estadual como objeto de in- 
teresse historiográfico. Nessa direção, se realiza uma análise crítica do bem-estar rural argentino na perspectiva histórica, circunscrito na primeira metade do século XX. Para isso, primeiro, se exploram a contribuição historiográfica produzida sobre esta temática, suas possibilidades analíticas y alguns vazios subsistentes. A continuação, se debate sobre os usos do rural na compreensão histórica das políticas sociais. Finalmente, se resume alguns progressos realizados na elucidação das experiências históricas do bem-estar rural na Argentina que podem ser uteis para uma discussão em andamento.

Palavras-chaves: bem-estar rural, políticas públicas, Estado, Historiografia social.

État et bien-être rural argentin lors de la première moitié du XXème siècle. Un problème historiographique

\section{Résumé}

Le but est celui d'avancer dans la construction du bien-être rural d'État comme objet d'intérêt historiographique. Dans ce sens, une analyse critique du bien-être rural dans une perspective historique est réalisée, circonscrit à la première moitié du XXème siècle. Pour ce faire, tout d'abord sont explorés les apports historiographiques produits autours de cette thématique, leurs possibilités analytiques et quelques vides subsistants. Ensuite, un débat est déroulé à propos des usages de ce qui est rural dans la compréhension historique des politiques sociales. Enfin certaines avancées sont résumées dans l'élucidation des expériences historiques du bien-être rural d'État en Argentine qui peuvent être utiles pour une discussion en gestation.

Mots clés: Bien-être rural, politiques publiques, État, historiographie sociale.

\section{INTRODUCCIÓN}

En los últimos años, a partir de distintos enfoques teóricos y estudios de caso, ha cobrado relevancia la discusión sobre el desarrollo rural y sus componentes. En general, los debates han girado en torno al modelo y los instrumentos que, desde la década de 1990, son capaces de mejorar las condiciones de vida de los sectores más pobres y rezagados de zonas rurales. En este sentido, un proclamado nuevo paradigma, denominado "desarrollo territorial rural", preocupado por la proyección espacial de 
las interacciones políticas, sociales, económicas e institucionales, propone estrategias de descentralización, de democracia participativa y de organización de los actores de la sociedad civil. Cabe señalar que en este paradigma el Estado es pensado como un sujeto subordinado en las discusiones y las posibles propuestas de solución.

Esa mirada anclada en tiempos contemporáneos nos ha inspirado a reflexionar sobre el derrotero histórico de las políticas sociales en los espacios rurales, las maneras en que se distribuyeron bienes y servicios públicos en el pasado y de qué modo estos impactaron en las condiciones de vida de la población. En otras palabras, la inmanencia de las controversias sobre el rol del Estado en el desarrollo rural nos ha impulsado a reflexionar sobre cómo las agencias estatales dirimieron la distribución del bienestar entre las poblaciones de menor tamaño, en complejos y variados contextos socioeconómicos e institucionales. La presunción de historicidad de la agenda estatal rural nos induce a preguntarnos sobre el surgimiento de una "cuestión social rural", las áreas de intervención privilegiadas en pos de alcanzar el bienestar rural, los instrumentos de acción diseñados e instrumentados, los agentes elegidos para llevar a cabo la tarea, el despliegue territorial, el vínculo con los actores agrarios, los resultados alcanzados y cómo todos estos procesos fueron variando a lo largo del tiempo. En síntesis, nos lleva a cuestionarnos respecto a la viabilidad de una línea de investigación escasamente abordada por la comunidad académica argentina, en general, dedicada a reflexionar sobre las políticas sociales en los principales polos urbanos.

En pos de adentrarnos en este prometedor campo de estudios, en el siguiente artículo realizamos un análisis crítico del bienestar rural argentino en perspectiva histórica, ciñéndonos a la primera mitad del siglo XX etapa de configuración de una matriz estatal del bienestar rural en el país. Para ello, primero, repasamos los avances historiográficos producidos sobre la temática, sus posibilidades analíticas y algunos vacíos subsistentes. A continuación, reflexionamos sobre los usos de lo rural en la comprensión histórica de las políticas sociales. Finalmente, resumimos algunos avances que consideramos valiosos en la dilucidación de las experiencias históricas del bienestar rural y las políticas sociales en la Argentina. 


\section{LA HISTORIOGRAFÍA SOBRE EL BIENESTAR RURAL ARGENTINO}

A partir de los años ochenta, la historia social argentina fue protagonista de una acelerada carrera ascendente, momento en el que se multiplicaron las líneas de investigación, las perspectivas teóricas y las estrategias metodológicas. A los estudios clásicos sobre el sindicalismo argentino se sumarían temáticas relativas al mundo del trabajo, las condiciones de vida material, las experiencias familiares, femeninas e infantiles, las prácticas de sociabilidad, asociacionismo y ocio. Estas prolíficas incursiones implicarían el desarrollo de una verdadera tradición disciplinar en torno a los problemas de la "cuestión social", la "cuestión obrera" y las emergentes políticas sociales introducidas a instancias de los procesos de modernización, secularización, urbanización, industrialización y construcción estatal que sobrevinieron durante la primera mitad del siglo $\mathrm{XX}^{1}$. El problema del bienestar rural, sin embargo, quedó relegado entre las inquietudes intelectuales de ese campo historiográfico y, durante más de dos décadas, los historiadores sociales argentinos demostraron una vocación poco sistemática por el estudio de las modalidades de acceso al bienestar entre las poblaciones rurales, concentrando su interés en los espacios urbanos, en especial en ciudades grandes e intermedias ubicadas en la región pampeana. Lentamente, en los últimos quince años, este panorama ha comenzado a modificarse, con el despliegue de una mayor sensibilidad respecto a las coordenadas materiales y simbólicas que identificaron las experiencias de individuos, familias y colectivos en espacios rurales. Como consecuencia, nuevos estudios, publicaciones y eventos académicos han canalizado esta preocupación por conocer la "cuestión social", la "cuestión obrera" y las "políticas sociales" argentinas en remozadas versiones rurales.

1 Algunos textos clásicos de esta tradición son: Leandro Gutiérrez, "Condiciones de la vida material de los sectores populares en Buenos Aires: 1880-1914”, Revista de Indias No. 41 (Ene 1, 1981): 167202; Leandro Gutiérrez y Luis Alberto Romero, Sectores populares, cultura y política. Buenos Aires en las entreguerras (Buenos Aires: Siglo XXI Editores, 2007 [1995]), 216; Diego Armus (comp.), Mundo urbano y cultura popular. Estudios de historia social argentina (Buenos Aires: Sudamericana, 1990), 361; Eduardo Zimmermann, Los liberales reformistas. La cuestión social en la Argentina 1890-1916 (Buenos Aires: Ed. Sudamericana/Universidad de San Andrés, 1995), 250; Juan Suriano (comp.), La cuestión social en la Argentina 1870-1943 (Buenos Aires: Editorial La Colmena, 2000), 334. El libro compilado por Juan Suriano puede considerarse en alguna medida un parteaguas de esta tradición al incorporar dos trabajos dedicados a contextos rurales. Un capítulo a cargo de Celia Bravo sobre los trabajadores azucareros y otro de Enrique Mases dedicado a la "cuestión indígena" en el norte patagónico. 
Inicialmente, es posible afirmar que buena parte de los trabajos ligados al bienestar y a las políticas públicas en los espacios rurales argentinos ha sido estimulada por dimensiones relativas a cuestiones agrarias ${ }^{2}$. El desarrollo de la historia de la educación técnica patentiza esta tendencia. La vitalidad de los estudios obedece, en gran medida, al interés por los cambios y continuidades de los perfiles productivos y su explicación a partir de la conformación de saberes especializados en torno al agro, su transmisión y al rol del Estado en ese proceso ${ }^{3}$. Estas contribuciones, por cierto ricas, develan básicamente los mecanismos de articulación entre las políticas públicas y las dinámicas económicas, predominando una visión utilitarista del problema del bienestar.

Similar directriz se observa en el terreno de la política laboral, cuyo análisis ha sido traccionado por los interrogantes sobre el mercado de trabajo estacional emplazado en la región cerealera y en las economías del interior (como la azucarera, vitivinícola, algodonera o yerbatera) entre finales del siglo XIX y las primeras décadas del $\mathrm{XX}^{4}$. En esta línea, el conocimiento sobre el origen de los trabajadores migrantes, una de las bases políticas del peronismo, cimentó indagaciones sobre sus tradiciones sindicales y experiencias con la mediación estatal ${ }^{5}$. Este tema incentivó también explo-

$2 \mathrm{Al}$ respecto, puede agregarse que en el mismo discurso estatal el problema de la "justicia social" en el mundo rural en general era traducido también como el de la tierra y el trabajo. Alejandra Salomón, "El bienestar social rural en el discurso peronista. Buenos Aires, 1952-1955", en Miradas desde la Historia social y la Historia intelectual. América Latina en sus culturas: de los procesos independistas a la globalización, eds. Hugo Cancino et al. (Córdoba: CEH-UCC, 2012), 681-694.

3 Talía Gutiérrez, Educación, agro y sociedad: Políticas educativas agrarias en la región pampeana, 1897-1955 (Bernal: UNQ, 2007), 262; Florencia Rodríguez Vázquez, Educación y vitivinicultura. Formación de recursos humanos y generación de conocimientos técnicos en Mendoza (1890-1920) (Rosario: Prohistoria, 2012), 211.

4 Los abordajes sobre el problema azucarero y la legislación laboral muestran la trascendencia de la movilización obrera rural a comienzos del siglo XX. Celia Bravo, "Liberales, socialistas, Iglesia y patrones frente a la situación de los trabajadores en Tucumán”, en La cuestión social en Argentina, 1870-1943, comp. Juan Suriano (Buenos Aires: La Colmena, 2000), 31-61. Alejandra Landaburu, "Los industriales y el Departamento Provincial del Trabajo ante el conflicto obrero de 1919 en Tucumán", en La sociedad del trabajo. Las instituciones laborales en la Argentina (1900-1955), comps. Mirta Zaida Lobato y Juan Suriano (Buenos Aires: Edhasa, 2014), 85-104.

5 Roberto Korzeniewicz, "Las vísperas del peronismo. Los conflictos laborales entre 1930 y 1943", Desarrollo Económico 131 (1993): 323-354; Adrián Ascolani, El sindicalismo rural en la Argentina. De la resistencia clasista a la comunidad organizada (1928-1952) (Bernal: UNQ, 2009), 392. 
raciones sobre la lenta institucionalización de nuevas formas de resolución estatal del conflicto laboral agrario argentino a comienzos del siglo $\mathrm{XX}^{6}$.

Ahora bien, más allá de estos ricos aportes, no resulta del todo claro si la dependencia de los problemas del bienestar rural respecto a las cuestiones agrarias ha sido el resultado de las perspectivas historiográficas adoptadas, o si, por el contrario, puede considerarse más bien un sesgo nativo. En el último apartado retomaremos la trayectoria histórica de esta interdependencia entre las políticas sociales y las políticas agrarias.

Comparativamente, el estudio sobre las condiciones de reproducción en el mundo rural en la primera mitad del siglo XX ha propiciado menor atención que las temáticas agrarias. Esto aplica tanto a la situación de la vivienda como a las formas de acceso a la salud, la alimentación, los servicios públicos, el ocio y la educación general. Esa desatención contrasta con la aparente paradoja del crecimiento económico argentino. Las investigaciones sobre el cinturón cerealero o las economías regionales cuyanas muestran que la expansión material de la época, el incremento de la producción y la comercialización de los productos de la tierra no se volcaron automáticamente en el mejoramiento de las condiciones de vida de la población rural ${ }^{7}$. Los niveles de analfabetismo, las dificultades para acceder a prestaciones médicas, la mala calidad de los alimentos, la desprotección de la infancia, las condiciones de inseguridad en los campos y la precariedad de las comunicaciones se habrían prolongado durante décadas.

6 Luciano Barandiarán, "El accidente fatal del trabajador rural y la justicia en el centro de la provincia de Buenos Aires (1935-1947)", Res Gesta 51 (2014): s/p; Juan Manuel Palacio, La Paz del trigo. Cultura legal y sociedad local en el desarrollo agropecuario pampeano 1890-1945 (Buenos Aires: Edhasa, 2004), 226; Juan Manuel Palacio, "De la Paz a la discordia: El peronismo y la experiencia del Estado en la Provincia de Buenos Aires (1943-1955)”, Desarrollo Económico vol. 49, Nº 194 (2009): 221-246; Adrián Ascolani, El sindicalismo rural, 392. ; Noemí Girbal-Blacha, Vivir en los márgenes. Estado, políticas públicas y conflictos sociales. El Gran Chaco Argentino en la primera mitad del siglo XX (Rosario: Prohistoria, 2011), 172; Juan Manuel Palacio, "La justicia peronista: El caso de las Cámaras de arrendamientos y aparcerías rurales (1948-1955)”, Anuario IEHS 26 (2011): 75-99.

7 Una mirada amplia sobre el problema del bienestar en los espacios rurales que pondera variables como la vivienda, la alimentación, la educación y la salud, puede verse en Beatriz Moreyra y Fernando Remedi, "Las cosas de todos los días en los espacios rurales de Córdoba a comienzos del siglo XX”, Anuario IEHS 20 (2005): 263-310; Juan Manuel Cerdá, Condiciones de vida y vitivinicultura. Mendoza, 1870-1950 (Bernal: UNQ, 2011), 251 
Es en el área de la salud y la enfermedad en donde pueden reconocerse, sin embargo, algunas excepciones al silencio reinante sobre estos temas. En ese sentido, han sido objeto de indagación las reacciones de las dirigencias políticas a afecciones como el paludismo, el bocio, el chagas y la fiebre hemorrágica argentina en contextos socioeconómicos dispares ${ }^{8}$. En cada caso, los historiadores destacaron las dificultades de los dispositivos sanitarios argentinos para adaptarse a las necesidades y los rasgos específicos de las poblaciones rurales, en gran medida producto del ralentizado proceso de "puesta en valor de una población que, hasta entonces, permanecía ajena a las preocupaciones de las autoridades sanitarias". Para algunos investigadores, la tardía adopción en el país de la medicina tropical constituyó un buen indicio de la lejanía que a comienzos del siglo XX existía entre los médicos y la suerte de la población rural ${ }^{10}$. Tal distancia discrepa con la centralidad que por la misma época asumieron las enfermedades del mundo urbano ${ }^{11}$. En esos términos, resultan interesantes los abordajes que desplazan la mirada de los contornos urbanos hacia los rurales e impugnan las visiones más lineales respecto al proceso de medicalización producido en la sociedad argentina. Proponen, en cambio, una trayectoria más sinuosa, conflictiva y tardía para la consolidación de la práctica médica, la expansión de los servicios de salud y el mejoramiento de las condiciones sanitarias de la población ${ }^{12}$.

$8 \quad$ Adriana Álvarez, Entre muerte y mosquitos. El regreso de las plagas en la Argentina (siglos XIX y XX) (Buenos Aires: Biblos, 2010), 219; Graciela Agnese, Historia de la fiebre hemorrágica argentina. Imaginario y espacio rural (1963-1990) (Rosario: Prohistoria, 2010), 169; Simone Petraglia Kropf, "En busca de la Enfermedad del Brasil: los médicos del interior y los estudios sobre el Mal de Chagas (1935-1956)", en Historia de salud y enfermedad en América Latina siglos XIX y XX, eds. Adrián Carbonetti y Ricardo González Leandri (Córdoba: CEA, 2008), 147-183; María Silvia Di Liscia, "Relaciones peligrosas: sobre bocio, cretinismo e inferioridad (Argentina, 1870-1920)", en De normas y transgresiones. Enfermedad y crimen en América Latina (1850-1950), eds. Claudia Agostoni y Elisa Speckman (México: IIH-UNAM, 2005), 21-54.

9 Adriana Álvarez, Entre muerte y mosquitos... 69.

10 Sandra Caponi, "Trópicos, microbios y vectores", História, Ciências, Saúde. Manguinhos, vol. 9 (2002): 111-38.

11 María Silvia Di Liscia, "Relaciones peligrosas".

12 Adrián Carbonetti y Adriana Álvarez, Fragmentos de la historia de la salud en la Argentina rural (Villa María: Eduvim, 2013), 138; Ernesto Bohoslavsky y María Silvia Di Liscia, "La profilaxis del viento. Instituciones represivas y sanitarias en la Patagonia argentina, 1880-1940", Asclepio, LX (2) (2008): 187-206; María Silvia Di Liscia, "Dificultades y Desvelos de un Estado interventor. Instituciones, salud y sociedad en el Interior Argentino. La Pampa, 1930-1946", Anuario IEHS, 22 (2007): 187-206; María Silvia Di Liscia, "Itinerarios curativos. Saberes, terapias y prácticas médicas indígenas, populares y científicas (Región Pampeana, 1750-1910)" (Tesis Doctoral, Universidad Complutense de Madrid, 2000), 485. 
La educación, la familia, el género y la infancia en el mundo rural son otras de las temáticas que, si bien en forma menos sistemática, han recibido atención en la historiográfica argentina. Especialmente, se han efectuado progresos respecto a las políticas educativas y asistenciales estructuradas con la finalidad de educar, nacionalizar y proteger a niños y mujeres ${ }^{13}$. Estas exploraciones han puesto de manifiesto la magnitud del temprano esfuerzo de los distintos niveles estatales en la escolarización infantil y la instrucción femenina; sin por ello olvidar las debilidades, incoherencias e indefiniciones estatales y la imprescindible intervención de la sociedad civil en la instrumentación de esos servicios públicos ${ }^{14}$. De tal modo, se han diversificado las publicaciones que destacan el activismo de la sociedad civil en la articulación de diferentes respuestas a los problemas sociales, a través de organizaciones de beneficencia, mutualismo, sociedades de fomento, cooperadoras escolares y agrupaciones de diferente tipo ${ }^{15}$. Esto ha favorecido una mirada más reflexiva sobre la formación estatal y, a su vez, de las relaciones cooperativas, complementarias y conflictivas, que los agentes estatales entablaron con la población en la generación de políticas sociales.

13 Talía Gutiérrez, Educación, agro y sociedad; Talía Gutiérrez, "Actuar sobre la mujer de campo, empleando a la mujer misma como educadora. Una visión histórica del discurso ruralista, Argentina, 1920-1945”, en Cuestiones agrarias en Argentina y Brasil, coords. Noemí M. Girbal-Blacha y Sonia Regina Mendoça (Buenos Aires: Prometeo, 2007), 183-202; Alejandra de Arce, Mujeres, familia y trabajo. Chacra, algodón y caña en la Argentina (1930-1960) (Bernal: UNQ, 2016), 320; Yolanda de Paz Trueba, Mujeres y esfera pública. La campaña bonaerense entre 1880 y 1910 (Prohistoria: Rosario, 2010), 172; María Andrea Nicoletti, "Formar ciudadanos argentinos y católicos en la Patagonia Norte de los Territorios Nacionales: La Congregación Salesiana y las escuelas del Estado (1880-1950)", Boletín Americanista 72 (2016): 71-88.

14 Adrián Ascolani, "La escuela primaria rural en Argentina. Expansión, orientaciones y dificultades (1916-1932)", Revista Teias vol. 14, № 28 (2012), 309-324; Adrián Ascolani, "Ruralidad, analfabetismo y trabajo en la Argentina. Proyectos y acciones del consejo nacional de educación (1930-1940)", Cadernos de História da Educação, vol. 3, N 14 (2015), 853-877; María José Billorou, "Los comedores escolares en el interior argentino (1930-1940). Discursos, prácticas e instituciones para el 'Apoyo a los Escolares Necesitados"', en Las infancias en la historia argentina. Intersecciones entre prácticas, discursos e instituciones (1890 -1960), comps. Lucía Lionetti y Daniela Míguez (Rosario: Prohistoria, 2010), 155-174.

15 Lucía Lionetti, "Políticas sociales del Estado y la sociedad civil desde el cuerpo de la niñez pobre en la Argentina (1900-1940)", Anuario del CEH, No 9 (2009): 97-116; María José Billorou: "Los niños en escena. Las políticas de protección a la infancia en el Territorio Nacional de la Pampa (1920-1940)", en Tierra adentro... instituciones económicas y sociales en los Territorios Nacionales (1884-1951), comps. Andrea Lluch y Marisa Moroni, (Rosario: Prohistoria/Universidad Nacional de La Pampa, 2010), 141-162. 
Una rápida revisión de los trabajos relevados nos proporciona entonces algunas pistas respecto a cómo la construcción del bienestar rural ha implicado un proceso complejo, de una enorme variabilidad en el tiempo y en el espacio y en el que estuvieron involucrados diversos niveles estatales y organizaciones civiles. El desplazamiento de la mirada desde los contextos urbanos hacia los espacios rurales, menos integrados política y socioeconómicamente, cuestiona entonces algunos de los supuestos sobre las capacidades del Estado para territorializarse y la actuación de los agentes civiles en la generación de bienes públicos.

Con base en la revisión de las principales contribuciones producidas, es posible asimismo vislumbrar algunas limitaciones de la historiografía sobre el bienestar rural en general y el estatal en particular. Una de ellas es el desarrollo desigual del conocimiento sobre el conjunto del espacio rural argentino. Lo que sabemos sobre estas cuestiones en buena medida sigue concentrado en la región pampeana y en los Territorios Nacionales ${ }^{16}$. Si bien se han dado pasos decisivos para incursionar en otras áreas, algunas provincias de las regiones noroeste, noreste, cuyana e incluso mesopotámica están claramente subrepresentadas en la producción historiográfica. La concreción de diálogos más fluidos entre los estudiosos de esas diferentes latitudes permitiría efectuar ejercicios comparativos sobre las dinámicas políticas, sociales y económicas entre los distintos espacios regionales.

En segundo lugar, es cada vez más evidente la necesidad de ampliar la mirada más allá del mundo estrictamente rural, relevando las articulaciones con la historia urbana y periurbana. Por ejemplo, examinando cómo los patrones de política social reducen el aislamiento y el éxodo rural al mejorar las condiciones materiales de vida y favorecer la integración económica, social y cultural de los habitantes de los pueblos rurales con las zonas circundantes.

En tercer lugar, resulta imperioso el planteo de nuevos temas y problemas,

16 Eran unidades político-administrativas creadas a fines del siglo XIX y carentes de autonomía. A partir de 1950 adquirieron el estatus de provincias. 
tales como la pobreza, el consumo, la vivienda rural ${ }^{17}$, los servicios de infraestructura social subsidiados o gestionados por el Estado (electricidad, agua potable, saneamiento, control de inundaciones, telecomunicaciones, transportes, red vial).

Cuarto, sería enriquecedor partir de la premisa de que las condiciones de vida en el espacio rural no resultan asimilables a las del medio urbano. En general, los estudios sobre el bienestar asocian la pobreza a la presencia o ausencia de indicadores elaborados para las ciudades, una medida abstracta confeccionada técnicamente que no forzosamente coincide con los umbrales de satisfacción definidos por la población rural. Esto se debe a que dichos umbrales conllevan una dimensión cultural, además de material, en la que vale la pena ahondar ${ }^{18}$.

Quinto, es necesario eludir la tendencia a equiparar el bienestar con las políticas sociales, puesto que existen políticas públicas no estrictamente sociales que contribuyen enormemente a la mejora en las condiciones de existencia. Por ejemplo, la electrificación genera sinergias positivas en la calidad de vida de los hogares rurales y suele ser subestimada por las miradas urbano-céntricas. Asimismo, el buen estado de los medios de comunicación, como caminos y ferrocarriles, es determinante en el desenvolvimiento rural: garantiza una mejor accesibilidad a los mercados, escuelas y servicios de salud; mejora los ingresos y las oportunidades de empleo; y promueve la sociabilidad. Variables que juegan un papel cardinal en la mitigación de la pobreza y en la radicación rural. Estas cuestiones complejizan necesariamente el abordaje del desarrollo rural, de sus realidades, posibilidades y restricciones.

Finalmente, en lo que hace estrictamente a las políticas públicas y al bienestar rural, el análisis de las políticas sociales en contextos rurales demanda una revisión más sistemática de nuestra comprensión de los contextos materiales y simbólicos en las que se estructuraron y de

17 Aproximaciones a la temática aparecen en Anahí Ballent, Las huellas de la política. Vivienda, ciudad, peronismo en Buenos Aires, 1943-1955 (Buenos Aires: UNQ, 2005), 280.

18 Amartya Sen, Desarrollo y libertad (Buenos Aires: Planeta: 2000), 440. 
los condicionamientos y posibilidades que los mismos impusieron a la construcción de intervenciones estatales. De tal modo, como veremos en las siguientes páginas, es altamente probable que esta agenda de investigación se vea beneficiada por una mayor reflexión sobre los comportamientos sociales, su incidencia en la configuración de las estructuras estatales y en la edificación del poder en el medio rural.

\section{Por una Clave rural de la historia del bienestar en la Argen- TINA}

En este apartado nuestro objetivo es reflexionar sobre la especificidad de una mirada de la ruralidad para un historiador interesado por las políticas sociales. No es novedoso afirmar que la misma definición de lo rural entraña grandes dificultades debido a la polisemia que el término ha tenido dentro del campo académico ${ }^{19}$. La asociación de lo rural con el predominio casi absoluto de actividades agropecuarias y baja densidad poblacional otorgó por mucho tiempo una identificación que permitía despejar algunas ambigüedades del concepto, con base en datos observables y cuantificables estadísticamente ${ }^{20}$. Sobre esta premisa, el espacio rural era definido por sus propiedades objetivas, en función de las distancias geográficas y de su estructuración interna.

Desde fines del siglo XIX y hasta el fin de la Segunda Guerra Mundial, el modelo explicativo del funcionamiento del espacio rural se basó en un enfoque dicotómico, en el que el campo era delimitado por su opuesto, la ciudad, según la variación de tamaño, formas y relación del hombre con la naturaleza. Las tradiciones sociológicas fueron importantes en la construcción de dicha conceptualización, al imprimir un fuerte acento binario a los sistemas de clasificación de la realidad, entre los que se destacó naturalmente la antinomia entre el campo y la ciudad. El mundo, implícitamente, se imaginaba dividido estrictamente en dos partes, separadas

19 Ángel Paniagua Mazorra y Keith Hoggart, "Lo rural, ¿hechos, discursos o representaciones? Una perspectiva geográfica de un debate clásico”, Globalización y mundo rural, № 803 (2002): 61-71.

20 Paul Cloke, "An Index of Rurality for England and Wales", Regional Studies, 11 (1977): 37-46; Vicki Harrington y Dan O' Donoghue, "Rurality in England and Wales 1991: A Replication and Extension of the 1981 Rurality Index", Sociologia Ruralis, vol. 38, No 2 (1998): 178-203. 
y separables: lo urbano y lo rural, donde lo segundo se miraba desde lo primero $^{21}$. En la segunda posguerra, si bien continuó primando la visión antinómica, comenzó a cobrar fuerza la idea de conexión entre ambos espacios. Se hizo referencia entonces al continuum, destacándose que los procesos rurales eran una continuidad espacial de los procesos urbanos; aunque los primeros siguieran siendo evocados en relación a los segundos, y no desde su propia dinámica.

Frente a las visiones cuantitativas que tuvieron peso en el pasado, pero también persisten en la actualidad, en la década del noventa otra vertiente introdujo una novedad: lo rural como una "construcción social" fundamentada en representaciones que orientan comportamientos y conduc$\operatorname{tas}^{22}$. El constructivismo como acercamiento alternativo al estudio del ámbito rural ha dado lugar a que el mismo fuera apreciado en todo su dinamismo, con sus propias singularidades y matices; y no como una entidad fija, cerrada, inmóvil y socialmente homogénea. Al respecto, es valioso el aporte de Raymond Williams, quien consideraba al campo no como una categoría sociológica, sino como un espacio cultural, construido por operaciones simbólicas que remiten a un imaginario social ${ }^{23}$. De esta manera, atribuía importancia a lo cultural como un elemento configurador de las relaciones sociales. Ello encierra una notable visión plural de la sociedad rural, donde cada grupo puede generar su propia representación espacial. Además, conduce a un enriquecimiento temático ligado a estilos de vida, visiones y valores.

A nuestro criterio, los estudios históricos sobre el mundo rural pueden beneficiarse particularmente de este abordaje que estimula la percepción de que el medio rural no se circunscribe al agro y que la comprensión de su dinámica exige el análisis de las representaciones sociales y las interacciones entre distintos actores. En este sentido, lo rural se asienta en la producción subjetiva del espacio por parte de quienes vivieron allí y, a la

21 Carlos Reboratti, "Los mundos rurales", en Población y bienestar en la Argentina del primero al segundo centenario, comp. Susana Torrado (Buenos Aires: Edhasa, 2007), 87.

22 Michel Blanc, "La ruralité: diversité des aproches", Économie rurale, $N^{\circ} 242$ (1997): 5-12. Keith Hoggart, "Let's do Away with Rural", Journal of Rural Studies, $\mathrm{N}^{\circ} 6$ (1990): 245-257.

23 Raymond Williams, El campo y la ciudad (Buenos Aires: Paidós, 2001). 410. 
vez, en prácticas políticas y culturales específicas. Esta aproximación invita a pensar el modo en que la configuración social del espacio influye en la identidad. Al respecto, Maria de Nazareth Baudel Wanderley propone dos elementos diferenciadores del espacio rural: la ocupación de un territorio con formas de dominación sustentadas en el uso y la tenencia de la tierra y de otros recursos naturales, y como lugar de vida, lo que otorga una identidad $^{24}$.

Esta focalización introduce otra cuestión: la historicidad. Como plantea Raymond Williams, el campo y la ciudad son realidades históricas variables, tanto en sí mismas como en las relaciones que mantienen entre sí, existiendo además muchos tipos de organizaciones intermedias y formaciones sociales y físicas ${ }^{25}$. El mundo rural detenta particularidades históricas, sociales, culturales, dotadas de una realidad propia, inclusive en su relación con el mundo urbano. Es decir, el tipo de relación social que caracteriza la ruralidad depende, en buena medida, del grado de articulación entre los habitantes rurales y aquellos de concentraciones urbanas. A su vez, estos grados de integración se corresponden con el acceso a servicios (educación, transportes, salud, red vial), a los mercados, al Estado, etc.; y condicionan el funcionamiento de la sociedad, la economía y la política locales. En esta línea, el estudio empírico de los sujetos, sus redes y sus organizaciones se vuelve absolutamente crucial para aprehender situaciones localizadas témporo-espacialmente.

Estas reflexiones sobre el mundo rural nos proporcionan dos claves centrales para introducirnos en las lógicas del bienestar estatal en los espacios rurales de comienzos del siglo XX. Primero, debemos tomar nota de que se trata de territorios con una densidad poblacional relativamente baja (si bien enmarcada dentro de una gran variabilidad de situaciones, pero claramente diferenciada de la urbana), una intensa relación con el medio natural (ya sea a través del uso de los recursos y servicios naturales para la producción agropecuaria como por su aprovechamiento en otro tipo

24 Maria de Nazareth Baudel Wanderley, "A ruralidade no Brasil moderno. Por um pacto social pelo desenvolvimento rural”, en ¿Una nueva ruralidad en América Latina?, comp. Norma Giarracca (Buenos Aires: CLACSO, 2001), 31-44.

25 Raymond Williams, El campo y la ciudad, 357. 
de actividades) y redes territoriales que articulaban los ámbitos dispersos y los centros poblados de diferente tamaño, estando estos, según era la situación, fuertemente integrados al medio rural ${ }^{26}$.

Segundo, lo rural comprendía un tipo de relaciones sociales con un fuerte componente personal. Sobre la base de vigorosas relaciones familiares y vecinales es posible identificar algunas particularidades del medio rural de la primera mitad del siglo XX, tales como la relevancia de la figura del padre, la confianza/desconfianza, la familia y el compromiso con la vida local. Por otro lado, este tipo particular de relaciones sociales, que podían derivar tanto en colaboración como en conflictos, generaba, a su vez, un enérgico control social por parte de la comunidad. Ello abre un rico campo de exploración sobre experiencias y áreas de conocimiento no referenciadas estrictamente dentro de las líneas más tradicionales de la historiografía urbano-céntrica.

\section{Algunos supuestos para un Debate en Ciernes}

Como señalamos en la introducción, en el estado de los estudios sobre el bienestar rural en la Argentina aún persiste un conjunto muy amplio de preguntas pendientes: ¿La problemática del bienestar en los espacios rurales emergió como temática específica en la agenda estatal durante esa época? Si así fue, ¿en qué medida respondió a las necesidades y demandas de los destinatarios y no fue una réplica de las intervenciones públicas en los espacios urbanos? ¿Qué rasgos de los contornos rurales asumieron centralidad en su desarrollo? ¿Cuáles fueron las modalidades de intervención estatal? ¿Qué niveles estatales accionaron en forma predominante?

La revisión de la bibliografía detallada previamente, de la literatura teórica reseñada y de los trabajos que componen este dossier proporcionan algunas pistas sobre estos interrogantes y ponen de relieve la riqueza de una línea de estudios abocada a las modalidades en las que en el pasado se ha

26 Hortencia Castro y Carlos Reboratti, "Revisión del concepto de ruralidad en la Argentina y alternativas posibles para su redefinición”, Serie Estudios e Investigaciones $N^{\circ} 15$, Secretaría de Agricultura, Ganadería, Pesca y Alimentos, Buenos Aires (2007). 
producido, distribuido y/o usufructuado el bienestar social en los espacios rurales argentinos. Así pues, al momento nos es posible identificar tres grandes cuestiones que ameritan especial, aunque no exclusiva, ponderación a la hora de historizar el surgimiento y derrotero de una "cuestión social rural": la incidencia de los imaginarios sociales en las agendas públicas, la fragilidad organizativa de los actores rurales y los condicionantes coyunturales y estructurales presentes en el medio rural.

En primera instancia, la literatura sobre el tema permite intuir que, en la primera mitad del siglo XX, la concepción sobre lo rural generó importantes consecuencias a la hora de definir las políticas públicas. En un contexto en que el concepto de progreso estaba asociado al pasaje unilineal de lo tradicional a lo moderno, del agro a la industria, de lo rural a lo urbano, lo rural en general pareció subestimarse, ocupando un lugar residual, básicamente como un espacio productivo. El bienestar rural, en consecuencia, se erigía como una cuestión innombrada e incomprensible para buena parte de la dirigencia política argentina. En consonancia, las evidencias sugieren la escasez o inexistencia de programas oficiales específicos y continuos sobre los problemas de salud, trabajo, vivienda y asistencia social de la población rural. La discontinuidad y la falta de planeamiento a largo plazo habrían sido las marcas distintivas de tales políticas.

Que esa "cuestión social rural" resultara escasamente incorporada en los programas oficiales, en las agendas públicas y en las discusiones de las agencias técnicas - con exiguas excepciones ${ }^{27}$ - puede vincularse también con un segundo motivo. Esto es, al hecho de que las mismas poblaciones dispersas de la Argentina interior tuvieran dificultades para articular una "voz" audible y cohesionada capaz de obtener respuestas de algún tipo por parte de las autoridades públicas. En el caso de los trabajadores rurales, su inestabilidad y movilidad, así como el mayor aislamiento relativo, habrían contribuido a ello. En general, la aceptación de la inevitabilidad de sus condiciones de vida y/o la autogestión, en contextos con mayores niveles de ingresos como la pampa húmeda, habrían sido las actitudes más

27 Talía Violeta Gutiérrez, "La educación del colono pampeano en épocas de conflicto: entre la defensa de sus intereses y el control social, 1910-1920”, E.I.A.L., Vol. 16 No. 2 (2005): 85-110. 
comunes durante la primera mitad del siglo XX, a pesar de algunos estallidos puntuales (como el Grito de Alcorta, por ejemplo).

La inexistencia de un debate público sobre los problemas sociales de los espacios rurales y la generación de dispositivos eficaces pareció comenzar a ser subsanada a partir de los años treinta, momento en el que la mayoría de los trabajos sitúan un quiebre en la identificación y el tratamiento de los problemas sociales de la Argentina rural ${ }^{28}$. La centralidad de ese corte no es casual, sino que responde a una crisis del mundo agrario, que impulsó a la dirigencia política a esbozar respuestas a los desafíos generados por la supervivencia de un modo de vida y un proyecto de país atado a la producción agropecuaria ${ }^{29}$. En esos términos, el descubrimiento de la Argentina rural, de sus habitantes, necesidades y conflictos, fue de la mano de las migraciones internas, del despoblamiento del campo, de la crisis agraria, de la emergencia de endemias y de la pobreza como agentes que dañaban la fortaleza de la Nación.

Una etapa diferente se habría desplegado a partir de los años cuarenta, en tanto algunas contribuciones revelan una acción pública más sistemática sobre la población rural a partir de los gobiernos peronistas. Los intentos de aplicación del llamado Estatuto del Peón Rural y las políticas de sanitarismo dan cuenta de una preocupación por los problemas, demandas y conflictos de la Argentina rural, destinada a trastocar su marginalidad previa. Ahora bien, esos virajes no habrían implicado la erosión de la noción de que el crecimiento del país se sustentaba en la expansión de los sectores industriales y en la urbanización de la vida moderna. Asimismo, habría subsistido la tendencia a tratar los problemas rurales con una lógica urbana y a acompasar la agenda del bienestar social rural con la agenda productiva.

28 Esto avala las visiones que indican cómo en distintos espacios regionales resultó evidente que la "cuestión social" constituía también un problema rural. Noemí Girbal-Blacha, Vivir en los márgenes... 76 .

29 Anahí Ballent y Adrián Gorelik, "País urbano o país rural: la modernización territorial y su crisis", en Nueva Historia Argentina, crisis económica, avance del estado e incertidumbre política (1930-1943), dir. Alejandro Cattaruzza (Buenos Aires: Sudamericana, 2001), 143-200. 
Varios de los dispositivos que comenzaron a implementarse sobre los espacios rurales desde los años treinta mostraron la permanencia de la inconmensurabilidad de esta dimensión territorial en la reflexión de las autoridades nacionales y provinciales y en sus elencos técnicos. No fue raro que las políticas diseñadas para resolver cuestiones socialmente conflictivas como la salud y la asistencia social fueran hegemonizadas por definiciones y soluciones producidas para los espacios urbanos concentrados. Además, las políticas sociales parecieron instituirse a partir de un complejo conjunto de relaciones, intereses e ideas que se presentaban en función de reclamos de "justicia social", pero respondían más a necesidades de la estructura productiva.

Este punto nos conduce a la tercera cuestión que queremos remarcar: los condicionantes. A pesar de algunos grandes esfuerzos, las cifras y diagnósticos indican fuertes brechas entre regiones y sectores de la población rural. Problemas básicos, que suelen ser minimizados o no suficientemente dimensionados desde un prisma urbano -como el trabajo infantil o la precariedad de las comunicaciones-, incidieron en el alcance efectivo de las políticas de bienestar rural. Esas limitaciones, tanto coyunturales como estructurales (financieras, técnico-burocráticas, legales, políticas, sociales), entraron en colisión directa con muchos de los instrumentos con los que se pretendió mejorar la calidad de vida rural. La compleja institucionalización de las pocas políticas públicas existentes puede atribuirse en buena medida a las escasas capacidades estatales, pero también a la débil legitimidad de las autoridades, la importancia de las relaciones personales y la resistencia de la población.

Diversos agentes, como los maestros, policías, jefes políticos, autoridades municipales e incluso particulares, habrían corporizado al Estado en los espacios rurales de comienzo de siglo. Sin embargo, en los contornos rurales su legitimidad, instrumentos y saberes dependieron tanto de la esfera estatal como de la movilización de recursos y principios de valoración construidos localmente, tal como hemos mencionado en el anterior apartado. En territorios con una baja densidad de población relativa, lo rural comprendía entonces un tipo de relaciones sociales en el que predominaba un componente personal sustentado en vínculos vecinales y parentales 
entre una parte significativa de los habitantes. Confirma así, lo dicho por Christophe Albaladejo, quien destaca la centralidad que hasta los años sesenta asumieron dichos lazos en las sociedades rurales pampeanas ${ }^{30}$. Dicha proyección explica la profundidad de los compromisos, el carácter interpersonal de los tratos, el estilo a veces paternalista del liderazgo y el tratamiento discrecional de ciertos temas. Como consecuencia, la vulnerabilidad de las políticas sociales en esos contextos habría obedecido tanto a la reducida penetración de las agencias oficiales como a la mediación de los responsables de su aplicación, cuyos intereses personales y redes sociales condicionaban la aplicación de las normativas.

La instrumentación de políticas sociales rurales también habría debido franquear difíciles procesos de construcción de acuerdos y legitimidad entre los diversos actores agrarios. Esta realidad se exacerba, por ejemplo, en el caso de las economías azucareras del norte argentino, en donde los dueños de los ingenios concentraban el poder económico y político, constriñendo las posibilidades de movilización sindical y política e imponiendo a las nuevas reglas laborales un ritmo de avance al compás de las contiendas políticas. Por otro lado, una mirada más amplia permite vislumbrar la participación de los empresarios privados en la distribución del bienestar, y cómo su actuación definió derechos, atribuciones y responsabilidades entre los sujetos implicados ${ }^{31}$. La delimitación de las "reglas de juego" de la redistribución social excedió, por cierto, al Estado.

\section{A Modo De CIERRE}

El disparador de este artículo ha sido la incertidumbre respecto a las especificidades y las potencialidades de un campo de estudio débilmente desarrollado en la historiografía argentina: el bienestar social rural. Desan-

30 Christophe Albaladejo, "De la pampa agraria a la pampa rural: la reconstrucción de las "localidades" y la invención del "desarrollo rural local”", Párrafos Geográficos, vol. 5, No 1 (2006): 33.

31 Tradicionalmente los ingenios azucareros implementaron discrecionalmente medidas asistenciales para sus trabajadores (de carácter educativo, sanitario y asistencial) como resultado de su utilitarismo como medio de control de la mano de obra, de la influencia del catolicismo social y de una perspectiva paternalista de las relaciones laborales. Alejandra Landaburu, "Paternalismo empresarial y condiciones de vida en los ingenios azucareros tucumanos. Fines del siglo XIX y principios del XX", Historia Regional, No 33 (2015): 27-49. 
dando el camino, tenemos el convencimiento de que el impacto del giro constructivista, el desarrollo de una reflexión más sistemática sobre los juegos de escala y la emergencia de los estudios sobre el Estado han creado un clima favorable para pensar los problemas de la "cuestión social" y el acceso al bienestar estatal en clave rural.

Para abordar esa historia del mundo rural, en primera instancia, es fundamental visibilizar la heterogeneidad como un rasgo intrínseco de la formación estatal y la provisión del bienestar a lo largo de Argentina. Todo intento por eludir la complejidad favorece miradas uniformadoras que obturan el conocimiento de las experiencias pretéritas. La diversidad de escenarios, de factores operantes, actores y condicionantes materiales y simbólicos constituye una norma para pensar los desajustes sociales y las respuestas que estos motorizaron a lo largo del tiempo. Las realidades pampeanas, norteñas, territorianas y cuyanas aquí exploradas son concluyentes a la hora de demostrar ese variopinto universo del bienestar rural argentino.

Ahora bien, captar la variabilidad histórica no implica renunciar al reconocimiento de regularidades y patrones comunes. Los espacios rurales y urbanos argentinos vivieron interconectados y afectados por procesos de escala regional, nacional e internacional que acompasaron los ritmos y la orientación de las transformaciones estatales y sociales. Las posibilidades de construir una periodización de la reforma de las agendas públicas y la estructuración de acciones estatales apuntan en este sentido y abonan el camino para próximos descubrimientos.

Finalmente, resulta imprescindible una aproximación más sistemática destinada a develar la compleja matriz social existente en los espacios rurales y cómo incidió en el acceso al bienestar. Una mirada atenta muestra que la sociedad civil, la sociedad política y los aparatos estatales han mantenido fluidas, continuas y estrechas relaciones en la identificación y resolución de los desajustes sociales. En particular, por lo que hemos analizado, es posible aseverar que las políticas sociales en los espacios rurales parecieron depender más de los incentivos producidos por las estructuras agrarias 
y las estrategias de actores locales que de proyectos, medidas y sujetos emanados de los centros de poder estatales. Una mirada de "abajo hacia arriba" y desde "los márgenes hacia el centro" puede constituir una fructífera estrategia analítica para enfrentar una agenda del bienestar rural en el futuro.

\section{Bibliografía}

Aelo, Oscar. El peronismo en la provincia de Buenos Aires 1946-1955. Caseros: Eduntref, 2012.

Agnese, Graciela. Historia de la fiebre hemorrágica argentina. Imaginario y espacio rural (1963-1990). Rosario: Prohistoria, 2010.

Albaladejo, Christophe. "De la pampa agraria a la pampa rural: la reconstrucción de las "localidades" y la invención del "desarrollo rural local””. Párrafos Geográficos, vol. 5, No 1 (2006): 27-52.

Álvarez, Adriana. Entre muerte y mosquitos. El regreso de las plagas en la Argentina (siglos XIX y XX). Buenos Aires: Biblos, 2010.

Armus, Diego (comp.). Mundo urbano y cultura popular. Estudios de historia social argentina. Buenos Aires: Sudamericana 1990.

Ascolani, Adrián. "La escuela primaria rural en Argentina. Expansión, orientaciones y dificultades (1916-1932)". Revista Teias, vol. 14, N 28 (2012): 309-324.

Ascolani, Adrián. "Ruralidad, analfabetismo y trabajo en la Argentina. Proyectos y acciones del consejo nacional de educación (1930-1940)". Cadernos de História da Educação, vol. 3, Nº 14 (2015): 853-877

Ascolani, Adrián. El sindicalismo rural en la Argentina. De la resistencia clasista a la comunidad organizada (1928-1952). Bernal: UNQ, 2009.

Bacolla, Natacha. "Política, administración y gestión en el peronismo santafecino, 1946-1955”. En La invención del peronismo en el interior del país, editado por Darío Macor y César Tcach. Santa Fe: UNL, 2003, 111-162.

Ballent, Anahí y Adrián Gorelik. "País urbano o país rural: la modernización territorial y su crisis”. En Nueva Historia Argentina, crisis económica, avance del estado e incertidumbre política (1930-1943), dirigido por Alejandro Cattaruzza. Buenos Aires: Sudamericana, 2001, 143-200. 
Ballent, Anahí. Las huellas de la política. Vivienda, ciudad, peronismo en Buenos Aires, 1943-1955. Buenos Aires: UNQ, 2005.

Barandiarán, Luciano. "El accidente fatal del trabajador rural y la justicia en el centro de la provincia de Buenos Aires (1935-1947)". Res Gesta 51 (2014): s/p.

Baudel Wanderley, Maria de Nazareth. "A ruralidade no Brasil moderno. Por um pacto social pelo desenvolvimento rural". En ¿Una nueva ruralidad en América Latina?, compilado por Norma Giarracca. Buenos Aires: CLACSO, 2001, 31-44.

Berrotarán, Patricia, Aníbal Jáuregui, y Marcelo Rougier. Sueños de bienestar en la Nueva Argentina. Estado y políticas públicas durante el peronismo, 1946-1955. Buenos Aires: Imago Mundi, 2004.

Berrotarán, Patricia. Del plan a la planificación. El estado durante la época peronista. Buenos Aires: Imago Mundi, 2003.

Billorou, María José. "Los comedores escolares en el interior argentino (1930-1940). Discursos, prácticas e instituciones para el 'Apoyo a los Escolares Necesitados”. En Las infancias en la historia argentina. Intersecciones entre prácticas, discursos e instituciones (1890-1960), compilado por Lucía Lionetti y Daniela Míguez. Rosario: Prohistoria, 2010, 155-174.

Billorou, María José. “Los riñas en escena. Las políticas de protección a la infancia. en el territorio, nacional de la pampa (1920-1940). En tierra adentro Instituciones ecónomicas y locales en los territorios nacionales (1884-1951), compilado por Andrea Lluch y Marisa Moroni Rosario: Prohistoria Universidad Nacional de la Pampa, 2010, 141-162.

Blanc, Michel. "La ruralité: diversité des aproches". Économie rurale, $\mathrm{N}^{\circ}$ 242 (1997): 5-12.

Bohoslavsky, Ernesto y María Silvia Di Liscia. "La profilaxis del viento. Instituciones represivas y sanitarias en la Patagonia argentina, 18801940”, Asclepio, LX (2) (2008): 187-206.

Bravo, Celia. "Liberales, socialistas, Iglesia y patrones frente a la situación de los trabajadores en Tucumán”. En La cuestión social en Argentina, 1870-1943, compilado por Juan Suriano. Buenos Aires: La Colmena, 2000, 31-61.

Campione, Daniel. Prolegómenos del peronismo. Los cambios en el Estado Nacional: 1943-1946. Buenos Aires: FISyP/Manuel Suárez, 2003. 
Caponi, Sandra. "Trópicos, microbios y vectores”, História, Ciências, Saúde. Manguinhos, vol. 9 (2002): 111-38.

Carbonetti, Adrián y Adriana Álvarez. Fragmentos de la historia de la salud en la Argentina rural. Villa María: Eduvim, 2013.

Castro, Hortencia y Reboratti, Carlos. "Revisión del concepto de ruralidad en la Argentina y alternativas posibles para su redefinición”. Serie Estudios e Investigaciones No 15, Secretaría de Agricultura, Ganadería, Pesca y Alimentos, Buenos Aires (2008).

Cerdá, Juan Manuel. Condiciones de vida y vitivinicultura. Mendoza, 18701950. Bernal: UNQ, 2011.

Cloke, Paul. "An Index of Rurality for England and Wales". Regional Studies, 11 (1977): 37-46.

de Arce, Alejandra. Mujeres, familia y trabajo. Chacra, algodón y caña en la Argentina (1930-1960). Bernal: UNQ, 2016.

de Paz Trueba, Yolanda. Mujeres y esfera pública. La campaña bonaerense entre 1880 y 1910. Prohistoria: Rosario, 2010.

Di Liscia, María Silvia. "Dificultades y Desvelos de un Estado interventor. Instituciones, salud y sociedad en el Interior Argentino. La Pampa, 1930-1946". Anuario IEHS, 22 (2007): 187-206.

Di Liscia, María Silvia. "Imaginarios y derroteros de la salud en el interior argentino. Los Territorios Nacionales (fines del siglo XIX y principios del XX)". Entrepasados 33 (2008).

Di Liscia, María Silvia. "Relaciones peligrosas: sobre bocio, cretinismo e inferioridad (Argentina, 1870-1920)". En De normas y transgresiones. Enfermedad y crimen en América Latina (1850-1950), editado por Claudia Agostoni y Elisa Speckman. México: IIH-UNAM, 2005. Di Liscia, María Silvia. "Itinerarios curativos. Saberes, terapias y prácticas médicas indígenas, populares y científicas (Región Pampeana, 17501910)". (Tesis Doctoral, Universidad Complutense de Madrid, 2000).

Girbal-Blacha, Noemí. Vivir en los márgenes. Estado, políticas públicas y conflictos sociales. El Gran Chaco Argentino en la primera mitad del siglo XX. Rosario: Prohistoria, 2011.

Gutiérrez, Leandro y Luis Alberto Romero. Sectores populares, cultura y política. Buenos Aires en las entreguerras. Buenos Aires: Siglo XXI Editores, 2007 [1995]. 
Gutiérrez, Leandro. "Condiciones de la vida material de los sectores populares en Buenos Aires: 1880-1914”. Revista de Indias 41 (Ene 1, 1981): 167-202.

Gutiérrez, Talía. "Actuar sobre la mujer de campo, empleando a la mujer misma como educadora. Una visión histórica del discurso ruralista, Argentina, 1920-1945”. En Cuestiones agrarias en Argentina y Brasil, coordinado por Noemí M. Girbal-Blacha y Sonia Regina Mendoça. Buenos Aires: Prometeo, 2007.

Gutiérrez, Talía. "La educación del colono pampeano en épocas de conflicto: entre la defensa de sus intereses y el control social, 19101920”. E.I.A.L., Vol. 16 № 2 (2005): 85-110.

Gutiérrez, Talía. Educación, agro y sociedad: Políticas educativas agrarias en la región pampeana, 1897-1955. Bernal: UNQ, 2007.

Harrington, Vicki y O' Dan Donoghue,. "Rurality in England and Wales 1991: A Replication and Extension of the 1981 Rurality Index". Sociologia Ruralis, vol. 38, No 2 (1998): 178-203.

Hirschegger, Ivana. "Alcance territorial de las políticas públicas peronistas en la provincia de Mendoza (Argentina), 1946-1955”. Apuntes, 66 (2010).

Hoggart, Keith. "Let’s Do Away with Rural”. Journal of Rural Studies,No 6 (1990): 245-257.

Korzeniewicz, Roberto. "Las vísperas del peronismo. Los conflictos laborales entre 1930 y 1943”. Desarrollo Económico 131 (1993): 323-354

Landaburu, Alejandra. "Paternalismo empresarial y condiciones de vida en los ingenios azucareros tucumanos. Fines del siglo XIX y principios del XX". Historia Regional, No 33 (2015): 27-49.

Landaburu, Alejandra. "Los industriales y el Departamento Provincial del Trabajo ante el conflicto obrero de 1919 en Tucumán”. En La sociedad del trabajo. Las instituciones laborales en la Argentina (19001955), compilado por Mirta Zaida Lobato y Juan Suriano. Buenos Aires: Edhasa, 2014.

Lionetti, Lucia. "Políticas sociales del Estado y la sociedad civil desde el cuerpo de la mujer pobre en la Argentina (1900-1940)". Amario del CEH No 9 (2009):97-116.

42 Lluch, Andrea y Marisa Moroni, (comps.). Tierra adentro...Instituciones económicas y sociales en los Territorios Nacionales (1884-1951). Rosario: Prohistoria, 2010. 
Moreyra, Beatriz y Fernando Remedi. "Las cosas de todos los días en los espacios rurales de Córdoba a comienzos del siglo XX”. Anuario IEHS 20 (2005): 263-310.

Moreyra, Beatriz. Cuestión social y políticas sociales en la Argentina. La modernidad periférica. Córdoba, 1900-1930. Bernal: UNQ, 2009.

Nicoletti, María Andrea. "Formar ciudadanos argentinos y católicos en la Patagonia Norte de los Territorios Nacionales: La Congregación Salesiana y las escuelas del Estado (1880-1950)". Boletín Americanista 72 (2016): 71-88.

Ortiz Bergia, María José. "El Estado en el interior nacional en la primera mitad del siglo XX. Aproximaciones historiográficas a un objeto en constante revisión”. Estudios Sociales del Estado, N 1 (2015): 59-85. Palacio, Juan Manuel. "De la Paz a la discordia: El peronismo y la experiencia del Estado en la Provincia de Buenos Aires (1943-1955)". Desarrollo Económico vol. 49, No 194 (2009): 221-246.

Palacio, Juan Manuel. "La justicia peronista: El caso de las Cámaras de arrendamientos y aparcerías rurales (1948-1955)". Anuario IEHS 26 (2011): 75-99.

Palacio, Juan Manuel. La Paz del trigo. Cultura legal y sociedad local en el desarrollo agropecuario pampeano 1890-1945. Buenos Aires: Edhasa, 2004.

Paniagua Mazorra, Ángel y Keith Hoggart,. "Lo rural, ¿hechos, discursos o representaciones? Una perspectiva geográfica de un debate clásico". Globalización y mundo rural, No 803 (2002): 61-71.

Petraglia Kropf, Simone. "En busca de la Enfermedad del Brasil: los médicos del interior y los estudios sobre el Mal de Chagas (1935-1956)". En Historia de salud y enfermedad en América Latina siglos XIX y XX, editado por Adrián Carbonetti y Ricardo González-Leandri. Córdoba: CEA, 2008, 147-183.

Piazzesi, Susana. Conservadores en provincia. El iriondismo santafesino 1937-1943. Santa Fe: UNL, 2009.

Prol, Mercedes. Estado, movimiento y partido peronista. La ingeniería institucional en Santa Fe, 1943-1955. Buenos Aires: Siglo XXI editores, 2012. Ramacciotti, Karina. La política sanitaria del peronismo. Buenos Aires: Biblos, 2009. 
Reboratti, Carlos. "Los mundos rurales". En Población y bienestar en la Argentina del primero al segundo centenario, compilado por Susana Torrado. Buenos Aires: Edhasa, 2007, 85-107.

Rodríguez Vázquez. Florencia. Educación y vitivinicultura. Formación de recursos humanos y generación de conocimientos técnicos en Mendoza (1890-1920). Rosario: Prohistoria, 2012.

Ruffini, Martha. La pervivencia de la República posible en los territorios nacionales. Poder y ciudadanía en Río Negro. Bernal: UNQ, 2007.

Salomón, Alejandra. "El bienestar social rural en el discurso peronista. Buenos Aires, 1952-1955”. En Miradas desde la Historia social y la Historia intelectual. América Latina en sus culturas: de los procesos independistas a la globalización, editado por Hugo Cancino et al. Córdoba: CEH-UCC, 2012, 681-694.

Sen, Amartya. Desarrollo y libertad. Buenos Aires: Planeta: 2000.

Suriano, Juan (comp.). La cuestión social en la Argentina 1870-1943. Buenos Aires: Editorial La Colmena, 2000.

Williams, Raymond. El campo y la ciudad. Buenos Aires: Paidós, 2001. Zimmermann, Eduardo A. Los liberales reformistas. La cuestión social en la Argentina 1890-1916. Buenos Aires: Ed. Sudamericana/Universidad de San Andrés, 1995.

Para citar este artículo: Salomón, Alejandra Laura y Ortiz Bergia, María 44 José. "Estado y bienestar rural argentino en la primera mitad del siglo XX. Un problema historiográfico”, Historia Caribe Vol. XII No. 31 (Julio-Diciembre 2017): 19-44. DOI: http://dx.doi.org/10.15648/hc.31.2017.2 